\title{
Weakly Bound States of Elementary Excitations in Graphene Superlattice in Quantizing Magnetic Field
}

\author{
Sergei V. Kryuchkov ${ }^{1,2}$ and Egor I. Kukhar'1 \\ ${ }^{1}$ Physical Laboratory of Low-Dimensional Systems, Volgograd State Socio-Pedagogical University, \\ V.I. Lenin Avenue, No. 27, Volgograd 400066, Russia \\ ${ }^{2}$ Department of Physics, Volgograd State Technical University, V.I. Lenin Avenue, No. 28, Volgograd 400005, Russia \\ Correspondence should be addressed to Sergei V. Kryuchkov; svkruchkov@yandex.ru
}

Received 21 October 2014; Revised 12 June 2015; Accepted 28 June 2015

Academic Editor: Sergei Sergeenkov

Copyright (c) 2015 S. V. Kryuchkov and E. I. Kukhar'. This is an open access article distributed under the Creative Commons Attribution License, which permits unrestricted use, distribution, and reproduction in any medium, provided the original work is properly cited.

\begin{abstract}
The spectrum of allowed energy of electron in graphene superlattice in the quantizing magnetic field is investigated. Such spectrum consists of number of so-called magnetic minibands. The width of these minibands depends on the superlattice barriers power and on the magnetic field intensity. The explicit form of electron spectrum is derived in the case of weak magnetic field. The possibility of electron-electron and electron-phonon bound states is shown. The binding energies of these states are calculated. The binding energy is shown to be the function of magnetic field intensity.
\end{abstract}

\section{Introduction}

The interactions of electrons with elementary excitations in crystal lattice have fundamental implications on properties of materials and lead to such many-body phenomena as superconductivity and charge-density waves. Such interactions take an unusual form in graphene [1-3] which can be described by the Dirac-like form of the effective Hamiltonian. A new area for investigations of chiral massless fermions is induced by discovery of this 2D material. So, interactions of Dirac electrons with elementary excitations which lead to the emergence of the bound states (BS) are under the intensive theoretical [4-7] and experimental study [8-10] last time.

Renormalization of Dirac spectrum due to interactions of electrons with lattice vibrations was obtained in [11-15]. Formations of polarons, plasmon-phonon complexes, and electron-hole pares are investigated in $[4,16-20]$, where BSs were shown to appear if energy of quasiparticles interaction exceeds a threshold value. In [19], the modification of Dirac spectrum near the threshold of optical phonon emission was studied. The spectrum characteristics of the electron-phonon quasiparticles (in particular, the electron-phonon binding energy versus the coupling parameter $g_{\text {el-ph }}$ ) were found in [19] within the theory, taking into account the singular vertex corrections beyond perturbation theory. Such corrections lead to the spectrum that remains below the optical phonon energy and corresponds to the electronphonon BS in comparison with that obtained within the Wigner-Brillouin perturbation theory [11-13].

Influence of various factors (geometry parameters, spinorbit interaction, charge impurities, etc.) on BS properties in graphene-based structures was studied in [4, 5, 21-27]. From an application technological point of view, the tunability of graphene electronic and optical properties by external fields is of particular importance [21, 28-32]. So, the effect of electric and magnetic fields on the properties of the BSs in graphene structures is of high interest among the researchers now [20, 21,33-35]. The influence of Landau level mixing on the energy of magnetoexcitons and magnetoplasmons in graphene was studied in [36], where dispersions of the excitations were shown to be changed by virtual transitions between Landau levels, caused by Coulomb interaction (electron-hole channel). In [20], the effect of the magnetic field on the electronphonon BSs was studied. The electron-phonon binding energy was shown to diverge at the resonant values of the magnetic field intensity. Such divergences corresponded to 
the electron-phonon hybrid states formed in the spectrum between the graphene Landau levels. These resonance states were obtained within the perturbation theory from the poles of the two-particle Green function and determine the structure of the magnetophonon resonance [37-39].

Presently, among the different graphene structures, the special attention is paid to graphene superlattices (GSLs) [40$44]$. Intensive investigations of electric and optical properties of GSL are explained by their different possible experimental and technological applications [31, 45-49]. Below we obtain the binding energies of electron-electron and electronphonon coupling in GSL in the quantizing magnetic field and determine the binding energy dependence on the magnetic field intensity and on the coupling parameters $g_{\text {el-el }}$ and $g_{\text {el-ph }}$.

\section{Electron Spectrum of GSL in Quantizing Magnetic Field}

In this section, we obtain the effective electron spectrum of GSL in quantizing magnetic field. We consider a GSL which is in the plane $x y$ (Figure 1). Near the Dirac point, the electron spectrum of GSL has the explicit form [40]:

$$
\varepsilon_{\mathrm{GSL}}(\mathbf{p})=\sqrt{u_{F}^{2} p_{x}^{2}+\Delta^{2} \sin ^{2} \frac{p_{y} d}{2 \hbar}}
$$

where $O y$ is the GSL axis (Figure 1), $u_{F}=v_{F} \sin \eta / \eta$ is the Fermi velocity along the axis $O x, \eta=V_{0} d / 4 \hbar v_{F}$ is the dimensionless power of SL barriers [40], $V_{0}$ is the barrier height, $d=2 \cdot 10^{-6} \mathrm{~cm}$ is the GSL period, $\Delta=2 \hbar v_{F} / d$, and $v_{F}=10^{8} \mathrm{~cm} / \mathrm{s}$ is the Fermi velocity in graphene. Linearized equation (equation analogous to the Dirac equation), which describes the motion of an electron in the GSL, takes the form $[50,51]$ :

$$
\left(u_{F} \sigma_{x} \widehat{\pi}_{x}+\Delta \sigma_{y} \sin \frac{\hat{\pi}_{y} d}{2 \hbar}\right) \psi=\varepsilon \psi .
$$

Here, $\boldsymbol{\sigma}=\left(\sigma_{x}, \sigma_{y}, \sigma_{z}\right)$ are the Pauli matrixes, $\psi$ is spinor function describing the electron states in GSL, $\widehat{\boldsymbol{\pi}}=\widehat{\mathbf{p}}+e \mathbf{A} / c$, $\widehat{\mathbf{p}}=-i \hbar\left(\partial_{x}, \partial_{y}\right), \mathbf{A}=(0, H x)$ is the vector potential, and $\mathbf{H}$ is the intensity of magnetic field which is supposed to be perpendicular to the GSL plane. Indeed, in the absence of magnetic field, the substitution of spinor for free charge carrier [52] into (2) gives the dispersion law (1).

In nonzero quantizing magnetic field $\left(\hbar v_{F} / \lambda_{H} \gg T\right.$, $\lambda_{H}=\sqrt{c \hbar / e H}$ is the Larmor radius, and $T$ is the temperature) we represent $\psi=e^{i k y} \chi$, where $\chi$ is spinor which is equal to

$$
\chi=\left(\begin{array}{l}
\chi_{1}(x) \\
\chi_{2}(x)
\end{array}\right)
$$

$\chi_{1}$ and $\chi_{2}$ describe electron states in the first and second graphene sublattice correspondingly. After acting on the spinor $\psi$ twice with the operator

$$
\widehat{H}_{\mathrm{GSL}}=u_{F} \sigma_{x} \widehat{\pi}_{x}+\Delta \sigma_{y} \sin \frac{\widehat{\pi}_{y} d}{2 \hbar},
$$

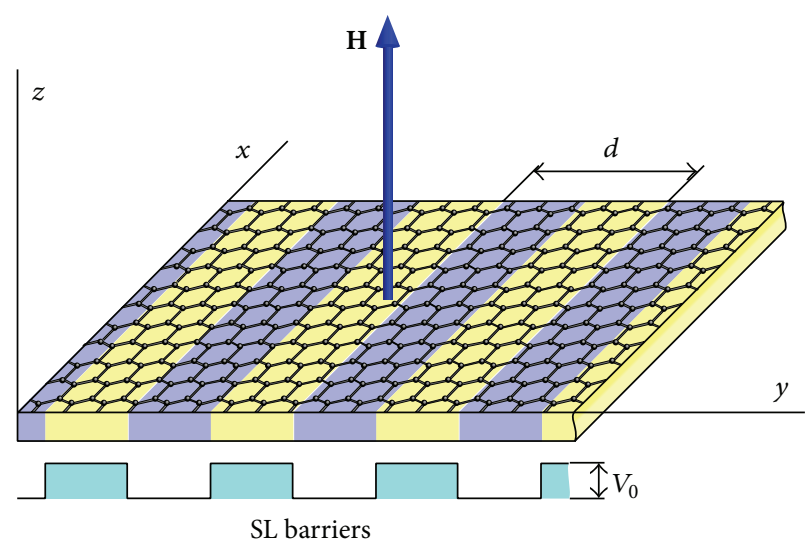

FIGURE 1: Schematic of the studied system.

using the next correlations $\widehat{H}_{\mathrm{GSL}}^{2} \psi=\varepsilon^{2} \psi, \sigma_{x} \sigma_{y}=-\sigma_{y} \sigma_{x}=$ $i \sigma_{z}$, and making a shift of the argument $k \lambda_{H}^{2}+x \rightarrow x$, we derive the following:

$$
-\hbar^{2} u_{F}^{2} \partial_{x}^{2} \chi+\Delta U(x) \chi=\left(\varepsilon^{2}-\Delta \Delta_{H} \sigma_{z}\right) \chi
$$

Here, we define $\Delta_{H}=2 \pi \hbar u_{F} / \Lambda_{H}, \Lambda_{H}=4 \pi \lambda_{H}^{2} / d$, and

$$
U(x)=\Delta \sin ^{2}\left(\frac{2 \pi x}{\Lambda_{H}}\right)-2 \Delta_{H} \sin ^{2}\left(\frac{\pi x}{\Lambda_{H}}\right) \sigma_{z} .
$$

To find the eigen values of $\varepsilon$ in (5) for the considered direction of vector $\mathbf{H}$ with respect to GSL axis, we use the method developed in [53]. It is easy to see from (6) that $U\left(x+\Lambda_{H}\right)=U(x)$ and therefore $\chi(x)$ satisfies the Bloch theorem. Thus, we can write

$$
\chi(x)=\sum_{n} e^{i n q \Lambda_{H}} \chi_{0}\left(x-n \Lambda_{H}\right)
$$

where $\chi_{0}$ is the eigenspinor of the next operator $\widehat{H}_{0}^{2}=$ $-\hbar^{2} u_{F}^{2} \partial_{x}^{2}+\Delta U(x)$ and corresponds to the electron state near the $n$th minimum of the function $U(x)$ [53]. Generally, the spectrum of allowed energy $\varepsilon$ consists of a number of minibands (magnetic minibands [53]). Having made some transformations, we derive

$$
\begin{aligned}
\varepsilon^{2} & -\left\langle\chi_{0}\left|\widehat{H}_{0}^{2}\right| \chi_{0}\right\rangle \\
& =4 \hbar^{2} u_{F}^{2} \chi_{0}^{+}\left(\frac{\Lambda_{H}}{2}\right) \partial_{x} \chi_{0}\left(\frac{\Lambda_{H}}{2}\right) \cos q \Lambda_{H} .
\end{aligned}
$$

For the weak magnetic fields $\left(\Delta_{H} \ll \Delta\right)$, the minima of $U(x)$ are separated by the wide barriers. In this case, the components of eigenspinor $\chi_{0}$ for the operator $\widehat{H}_{0}^{2}$ are close to the functions of the harmonic oscillator localized near the $n$th minimum of the function $U(x)$. Further, we consider that electron is in the lowest Landau level. Thus, we obtain instead (8)

$$
\varepsilon(q)=\sqrt{\Delta \Delta_{H}}-D_{H} \cos q \Lambda_{H},
$$


where

$$
D_{H}=8 \sqrt{\pi} \Delta \exp \left(-\frac{2 \pi^{2} \Delta}{\Delta_{H}}\right) .
$$

The width of allowed miniband is seen from (10) to depend on the GSL barriers power and on the magnetic field intensity.

\section{Emergence of the BS of Two Electrons in GSL}

Here, we show that in GSL with electron spectrum (9) in the presence of quantizing magnetic field the appearance of BS of two electrons is possible at arbitrarily weak repulsion between them. Equation that defines the dispersion law of the BS can be obtained by determining the poles of the twoparticle Green function [54]:

$$
\begin{aligned}
& \frac{g_{\mathrm{el}-\mathrm{el}}}{2 \pi} \int_{-\pi / \Lambda_{H}}^{+\pi / \Lambda_{H}} \frac{\mathrm{d} q}{E-\varepsilon_{1}(q-p / 2)-\varepsilon_{2}(q+p / 2)+i 0} \\
& \quad=1
\end{aligned}
$$

where $g_{\text {el-el }}$ is the effective coupling parameter of quasiparticles with dispersion lows $\varepsilon_{1}(q)$ and $\varepsilon_{2}(q)$ and $p$ is the momentum of BS. We use (11) in the case of electron-electron interaction in GSL in the presence of quantizing magnetic field. Equation (11) has undamped solutions if $g_{\text {el-el }}>0$ (repulsion) and $E>2 \sqrt{\Delta \Delta_{H}}+2 D_{H}$. After integration in (11), we obtain the dispersion law of BS:

$$
E(p)=2 \sqrt{\Delta \Delta_{H}}+\sqrt{\frac{g_{\text {el-el }}^{2}}{\Lambda_{H}^{2}}+4 D_{H}^{2} \cos ^{2} \frac{p \Lambda_{H}}{2}} .
$$

It is seen from (12) that electrons can bind if energy $E$ is close to the top of conduction band where effective masses are negative. This fact explains the possibility of electrons binding while $g_{\text {el-el }}>0$. Binding energy is

$$
E_{0}=\sqrt{\frac{g_{\mathrm{el}-\mathrm{el}}^{2}}{\Lambda_{H}^{2}}+4 D_{H}^{2}}-2 D_{H}
$$

For weak repulsion between electrons $\left(g_{\text {el-el }} \ll D_{H} \Lambda_{H}\right)$, the binding energy is proportional to square of $g_{\text {el-el }}: E_{0}=$ $g_{\text {el-el }}^{2} / 4 D_{H} \Lambda_{H}^{2}$. For intensive repulsion between electrons $\left(g_{\text {el-el }} \gg D_{H} \Lambda_{H}\right)$, we have $E_{0}=-2 D_{H}+g_{\text {el-el }} / \Lambda_{H}$. The binding energy dependence on the magnetic field intensity is shown in Figure 2 for different values of coupling parameter.

\section{Emergence of the Electron-Phonon BS in GSL}

Now, we obtain the possibility of electron-optical phonon BS in GSL in the quantizing magnetic field. The equation that determines the dispersion law of such BS is [54]

$$
\frac{g_{\mathrm{el}-\mathrm{ph}}}{2 \pi} \int_{-\pi / \Lambda_{H}}^{+\pi / \Lambda_{H}} \frac{\mathrm{d} q}{E-\hbar \omega_{0}-\varepsilon(q)+i 0}=1 .
$$

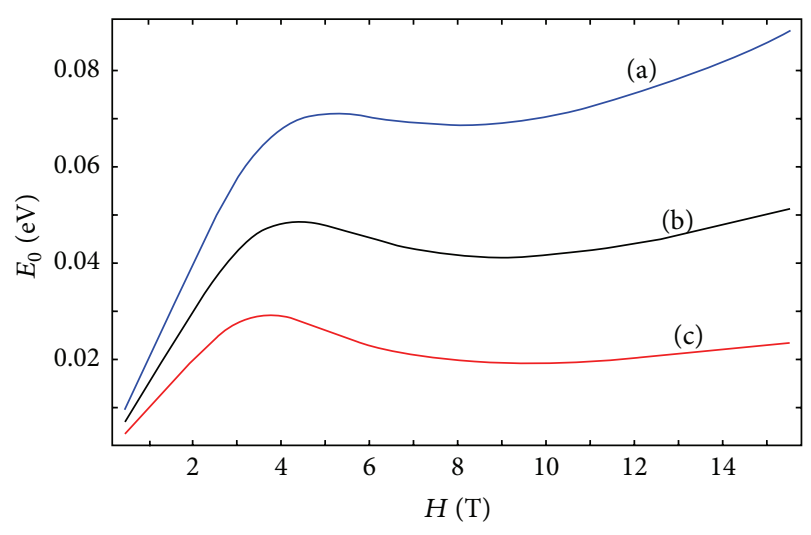

FIGURE 2: Binding energy $E_{0}$ versus the magnetic field intensity $H$. (a) $g_{\text {el-el }}=2 \hbar u_{F}$, (b) $g_{\text {el-el }}=1.5 \hbar u_{F}$, and (c) $g_{\text {el-el }}=\hbar u_{F}$.

Here, the coupling parameter $g_{\text {el-ph }}<0$ and corresponds to the attraction between electron and phonon and $\omega_{0}$ is the frequency of optical phonon which is assumed to be dispersionless. The undamped solution of (14) is possible if $E<\hbar \omega_{0}+\sqrt{\Delta \Delta_{H}}-D_{H}$. Dispersionless of optical phonon leads to the fact that electron-phonon BS appears at all values of quasiparticle momentum. In this case, the binding energy is

$$
E_{0}=\sqrt{\frac{g_{\mathrm{el}-\mathrm{ph}}^{2}}{\Lambda_{H}^{2}}+D_{H}^{2}}-D_{H} .
$$

In comparison with that obtained for electron-phonon coupling in graphene in the absence of magnetic field [19], the binding energy (15) does not exhibit exponential dependence on the coupling constant $g_{\mathrm{el}-\mathrm{ph}}$.

\section{Conclusion}

Above we have investigated the spectrum of allowed energy of electron in GSL in the presence of quantizing magnetic field $\left(\hbar v_{F} / \lambda_{H} \gg T\right)$. Generally, such spectrum consists of number of so-called magnetic minibands. The width of these minibands depends on the GSL barriers power and on the magnetic field intensity. For our calculations, we assume that electron is in the magnetic miniband which corresponds to the lowest harmonic oscillator state. The explicit form of electron spectrum in this case is (9). The formula (9) is adequate if magnetic field satisfies the next condition: $\Delta_{H} \ll$ $\Delta$, which is true up to $H=15 \mathrm{~T}$ (the power of GSL barriers is of $\eta \sim 3.136$ [40]). For such magnetic fields, temperature should not exceed $100 \mathrm{~K}$. Nitrogen boiling point is suitable to this end. Moreover, the upper limit of magnetic field can be increased by changing the power of GSL barriers $\eta$.

Thus, experimental measurements could confirm the suggested theory if one uses the GSL with the period $d=2$. $10^{-6} \mathrm{~cm}$ (typical value for superlattices $[41,45]$ ) and with the height of barrier $V_{0} \sim 0.392 \mathrm{eV}$ (in this case, $\eta \sim 3.136$ ). To this end, the GSL obtained by a sheet of graphene deposited on a banded substrate formed by periodically alternating layers of $\mathrm{SiC}$ and hexagonal $\mathrm{BN}$ is suitable one [41]. Another 
suitable structure could be obtained by periodical insertion of hydrogenated graphene (graphane [55]) between gapless graphene strips. For such material barrier height consists of $V_{0} \sim 2.7 \mathrm{eV}[41,55]$. If $V_{0} \sim 2.7 \mathrm{eV}$, then $\eta \sim 22$ and the condition $\Delta_{H} \ll \Delta$ will be also performed up to $H=15 \mathrm{~T}$.

Using (9), we have shown the possibility of electronelectron and electron-phonon BSs and have calculated the binding energy of these states, which is the function of magnetic field (Figure 2). In the case of electron-optical phonon coupling, the binding energy does not exhibit exponential dependence on the coupling constant $g_{\text {el-ph }}$ in comparison with that obtained for graphene in the absence of magnetic field [19]. Electron-phonon BS appears at all values of quasiparticle momentum. This feature is explained by the dispersionless of optical phonon.

In [20], the electron-phonon binding energy was shown to diverge in graphene at the resonant values of the magnetic field intensity. Such divergences correspond to the electronphonon hybrid states formed in the spectrum between the graphene Landau levels. As it is shown above in GSL electronphonon binding energy (15) has the finite maximum as well as electron-electron binding energy (Figure 2). It is obtained instead of divergence of the binding energy as it was for the case of single-layer graphene [20]. Finite maximum of the electron-electron and electron-phonon binding energy in GSL in quantizing magnetic field is due to the broadening of energy levels (Landau levels). Such broadening takes place if quantizing magnetic field is applied perpendicularly to the superlattice axis [53].

In the case of electron-electron coupling, BSs correspond to the Bose-Einstein statistics. At low temperatures (under the critical temperature), such electron system can form a superfluid gas [56]. The presence of additional potential in graphene in this case is essential for the following reason. Electrons can bind if energy $E$ is close to the top of conduction band of GSL where effective masses are negative (12). This fact explains the possibility of electrons binding while $g_{\text {el-el }}>0$. In the absence of additional potential (at the linear dispersion limit), there are no regions with negative effective masses. So, "repulsive" electron-electron interaction cannot form bound states in such limit.

\section{Conflict of Interests}

The authors declare that there is no conflict of interests regarding the publication of this paper.

\section{Acknowledgments}

The work was supported with the funding of the Ministry of Education and Science of the Russian Federation within the base part of the State task no. 2014/411 (Project code 522).

\section{References}

[1] E. H. Hwang and S. Das Sarma, "Dielectric function, screening, and plasmons in two-dimensional graphene," Physical Review B, vol. 75, no. 20, Article ID 205418, 2007.
[2] S. Gangadharaiah, A. M. Farid, and E. G. Mishchenko, "Charge response function and a novel plasmon mode in graphene," Physical Review Letters, vol. 100, no. 16, Article ID 166802, 2008.

[3] P. Burset, W. Herrera, and A. L. Yeyati, "Proximity-induced interface bound states in superconductor-graphene junctions," Physical Review B, vol. 80, no. 4, Article ID 041402(R), 2009.

[4] L. Brey and H. A. Fertig, "Elementary electronic excitations in graphene nanoribbons," Physical Review B, vol. 75, no. 12, Article ID 125434, 2007.

[5] K. S. Gupta and S. Sen, "Bound states in graphene," Modern Physics Letters A, vol. 24, no. 2, pp. 99-107, 2009.

[6] A. Bostwick, F. Speck, T. Seyller et al., "Observation of plasmarons in quasi-freestanding doped graphene," Science, vol. 328, no. 5981, pp. 999-1002, 2010.

[7] V. M. Apalkov and T. Chakraborty, "Gap structure of the Hofstadter system of interacting Dirac fermions in graphene," Physical Review Letters, vol. 112, no. 17, Article ID 176401, 2014.

[8] J. Yan, Y. Zhang, P. Kim, and A. Pinczuk, "Electric field effect tuning of electron-phonon coupling in graphene," Physical Review Letters, vol. 98, no. 16, Article ID 166802, 2007.

[9] S. Pisana, M. Lazzeri, C. Casiraghi et al., "Breakdown of the adiabatic Born-Oppenheimer approximation in graphene," Nature Materials, vol. 6, no. 3, pp. 198-201, 2007.

[10] A. Bostwick, T. Ohta, T. Seyller, K. Horn, and E. Rotenberg, "Quasiparticle dynamics in graphene," Nature Physics, vol. 3, no. 1, pp. 36-40, 2007.

[11] C.-H. Park, F. Giustino, M. L. Cohen, and S. G. Louie, "Velocity renormalization and carrier lifetime in graphene from the electron-phonon interaction," Physical Review Letters, vol. 99, no. 8, Article ID 086804, 2007.

[12] M. Calandra and F. Mauri, "Electron-phonon coupling and electron self-energy in electron-doped graphene: calculation of angular-resolved photoemission spectra," Physical Review B, vol. 76, no. 20, Article ID 205411, 2007.

[13] W.-K. Tse and S. Das Sarma, "Phonon-induced many-body renormalization of the electronic properties of graphene," Physical Review Letters, vol. 99, no. 23, Article ID 236802, 2007.

[14] D. M. Basko and I. L. Aleiner, "Interplay of Coulomb and electron-phonon interactions in graphene," Physical Review BCondensed Matter and Materials Physics, vol. 77, no. 4, Article ID 041409, 2008.

[15] E. Cappelluti and L. Benfatto, "Vertex renormalization in dc conductivity of doped chiral graphene," Physical Review B: Condensed Matter and Materials Physics, vol. 79, no. 3, Article ID 035419, 2009.

[16] T. Stauber and N. M. R. Peres, "Effect of Holstein phonons on the electronic properties of graphene," Journal of Physics: Condensed Matter, vol. 20, no. 5, Article ID 055002, 2008.

[17] V. M. Stojanović, N. Vukmirović, and C. Bruder, "Polaronic signatures and spectral properties of graphene antidot lattices," Physical Review B, vol. 82, no. 16, Article ID 165410, 2010.

[18] M. Jablan, M. Soljačić, and H. Buljan, "Unconventional plasmon-phonon coupling in graphene," Physical Review BCondensed Matter and Materials Physics, vol. 83, no. 16, Article ID 161409, 2011.

[19] S. M. Badalyan and F. M. Peeters, "Electron-phonon bound state in graphene," Physical Review B, vol. 85, no. 20, Article ID 205453, 2012.

[20] J. Zhu, S. M. Badalyan, and F. M. Peeters, "Electron-phonon bound states in graphene in a perpendicular magnetic field," Physical Review Letters, vol. 109, no. 25, Article ID 256602, 2012. 
[21] P. Recher, J. Nilsson, G. Burkard, and B. Trauzettel, "Bound states and magnetic field-induced valley splitting in gatetunable graphene quantum dots," Physical Review B, vol. 79, no. 8, Article ID 085407, 2009.

[22] S. Ryu, C. Mudry, C.-Y. Hou, and C. Chamon, "Masses in graphenelike two-dimensional electronic systems: topological defects in order parameters and their fractional exchange statistics," Physical Review B, vol. 80, no. 20, Article ID 205319, 2009.

[23] J. G. Xu, L. Wang, and M. Q. Weng, "Quasi-bound states and Fano effect in T-shaped graphene nanoribbons," Journal of Applied Physics, vol. 114, no. 15, Article ID 153701, 2013.

[24] D. Klöpfer, A. De Martino, and R. Egger, "Bound states and supercriticality in graphene-based topological insulators," Crystals, vol. 3, no. 1, pp. 14-27, 2013.

[25] M. M. Asmar and S. E. Ulloa, "Spin-orbit interaction and isotropic electronic transport in graphene," Physical Review Letters, vol. 112, no. 13, Article ID 136602, 2014.

[26] T. Iadecola, T. Neupert, and C. Chamon, "Topological gaps without masses in driven graphene-like systems," Physical Review B-Condensed Matter and Materials Physics, vol. 89, no. 11, Article ID 115425, 2014.

[27] M. M. Asmar and S. E. Ulloa, "Symmetry-breaking effects on spin and electronic transport in graphene," Physical Review B, vol. 91, no. 16, Article ID 165407, 2015.

[28] D. S. L. Abergel and T. Chakraborty, "Long-range coulomb interaction in bilayer graphene," Physical Review Letters, vol. 102, no. 5, Article ID 056807, 2009.

[29] V. M. Apalkov and T. Chakraborty, "Electrically tunable charge and spin transitions in Landau levels of interacting Dirac fermions in trilayer graphene," Physical Review B, vol. 86, no. 3, Article ID 035401, 2012.

[30] M. Zarenia, B. Partoens, T. Chakraborty, and F. M. Peeters, "Electron-electron interactions in bilayer graphene quantum dots," Physical Review B, vol. 88, no. 24, Article ID 245432, 2013.

[31] A. Pieper, R. L. Heinisch, G. Wellein, and H. Fehske, "Dotbound and dispersive states in graphene quantum dot superlattices," Physical Review B-Condensed Matter and Materials Physics, vol. 89, no. 16, Article ID 165121, 2014.

[32] E. Margapoti, P. Strobel, M. M. Asmar et al., "Emergence of photoswitchable states in a graphene-azobenzene-au platform," Nano Letters, vol. 14, no. 12, pp. 6823-6827, 2014.

[33] A. Pound, J. P. Carbotte, and E. J. Nicol, "Effects of electronphonon coupling on Landau levels in graphene," Physical Review B, vol. 84, no. 8, Article ID 085125, 2011.

[34] B. Skinner, B. I. Shklovskii, and M. B. Voloshin, "Bound state energy of a Coulomb impurity in gapped bilayer graphene," Physical Review B-Condensed Matter and Materials Physics, vol. 89, no. 4, Article ID 041405, 2014.

[35] P. Rakyta, E. Tóvári, M. Csontos, S. Csonka, A. Csordás, and J. Cserti, "Emergence of bound states in ballistic magnetotransport of graphene antidots," Physical Review B, vol. 90, no. 12, Article ID 125428, 2014.

[36] Y. E. Lozovik and A. A. Sokolik, "Influence of landau level mixing on the properties of elementary excitations in graphene in strong magnetic field," Nanoscale Research Letters, vol. 7, article 134, 19 pages, 2012.

[37] T. Ando, "Magnetic oscillation of optical phonon in graphene," Journal of the Physical Society of Japan, vol. 76, no. 2, Article ID 024712, 2007.
[38] M. O. Goerbig, J.-N. Fuchs, K. Kechedzhi, and V. I. Fal'ko, "Filling-factor-dependent magnetophonon resonance in graphene," Physical Review Letters, vol. 99, Article ID 087402, 2007.

[39] Y. Kim, J. M. Poumirol, A. Lombardo et al., "Measurement of filling-factor-dependent magnetophonon resonances in graphene using Raman spectroscopy," Physical Review Letters, vol. 110, no. 22, Article ID 227402, 2013.

[40] M. Barbier, P. Vasilopoulos, and F. M. Peeters, "Extra Dirac points in the energy spectrum for superlattices on single-layer graphene," Physical Review B, vol. 81, no. 7, Article ID 075438, 2010.

[41] P. V. Ratnikov, "Superlattice based on graphene on a strip substrate," JETP Letters, vol. 90, no. 6, pp. 469-474, 2009.

[42] J. A. Briones-Torres, J. Madrigal-Melchor, J. C. MartínezOrozco, and I. Rodríguez-Vargas, "Electrostatic and substratebased monolayer graphene superlattices: energy minibands and its relation with the characteristics of the conductance curves," Superlattices and Microstructures, vol. 73, pp. 98-112, 2014.

[43] J. C. W. Song, A. V. Shytov, and L. S. Levitov, "Electron interactions and gap opening in graphene superlattices," Physical Review Letters, vol. 111, no. 26, Article ID 266801, 2013.

[44] D. Bolmatov and C.-Y. Mou, "Graphene-based modulationdoped superlattice structures," Journal of Experimental and Theoretical Physics, vol. 112, no. 1, pp. 102-107, 2011.

[45] L. A. Falkovsky, "Optical properties of graphene and IV-VI semiconductors," Physics-Uspekhi, vol. 51, no. 9, pp. 887-897, 2008.

[46] T. G. Pedersen and J. G. Pedersen, "Transport in graphene antidot barriers and tunneling devices," Journal of Applied Physics, vol. 112, no. 11, Article ID 113715, 2012.

[47] J. G. Pedersen, T. Gunst, T. Markussen, and T. G. Pedersen, "Graphene antidot lattice waveguides," Physical Review BCondensed Matter and Materials Physics, vol. 86, no. 24, Article ID 245410, 2012.

[48] J. G. Pedersen and T. G. Pedersen, "Hofstadter butterflies and magnetically induced band-gap quenching in graphene antidot lattices," Physical Review B, vol. 87, no. 23, Article ID 235404, 2013.

[49] M. R. Thomsen, S. J. Brun, and T. G. Pedersen, "Dirac model of electronic transport in graphene antidot barriers," Journal of Physics: Condensed Matter, vol. 26, no. 33, Article ID 335301, 2014.

[50] V. Krueckl and K. Richter, "Bloch-Zener oscillations in graphene and topological insulators," Physical Review B, vol. 85, Article ID 115433, 2012.

[51] S. V. Kryuchkov and E. I. Kukhar, "Solitary electromagnetic waves in a graphene superlattice under influence of highfrequency electric field," Superlattices and Microstructures, vol. 70, pp. 70-81, 2014.

[52] P. Burset, A. L. Yeyati, L. Brey, and H. A. Fertig, "Transport in superlattices on single-layer graphene," Physical Review B, vol. 83, no. 19, Article ID 195434, 2011.

[53] A. G. Zhylich, "The energy spectrum of electrons and optical properties of the superlattice in a magnetic field," Physics of the Solid State, vol. 36, no. 3, pp. 792-805, 1994.

[54] L. P. Pitaevskii, "Weakly bound excitation states in a crystal," Journal of Experimental and Theoretical Physics, vol. 43, no. 2, pp. 382-388, 1976. 
[55] S. Lebègue, M. Klintenberg, O. Eriksson, and M. I. Katsnelson, "Accurate electronic band gap of pure and functionalized graphane from GW calculations," Physical Review BCondensed Matter and Materials Physics, vol. 79, no. 24, Article ID 245117, 2009.

[56] G. V. Uimin, L. A. Maksimov, and A. F. Barabanov, "Bound states of electrons (holes) on a square lattice and their superfluidity," JETP Letters, vol. 48, no. 5, pp. 312-316, 1988. 

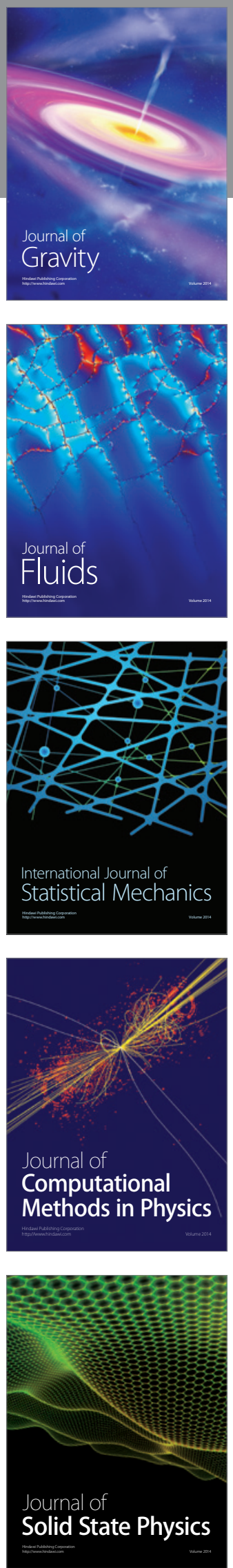

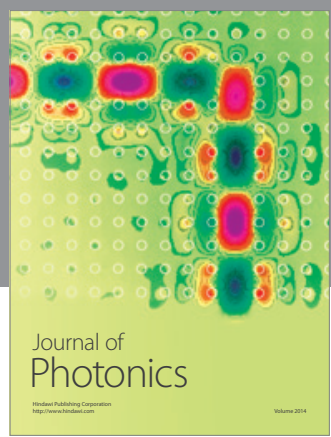

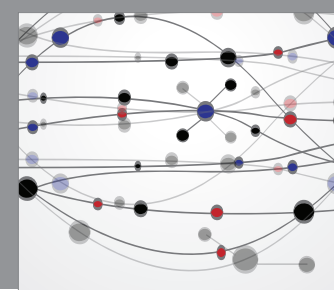

The Scientific World Journal

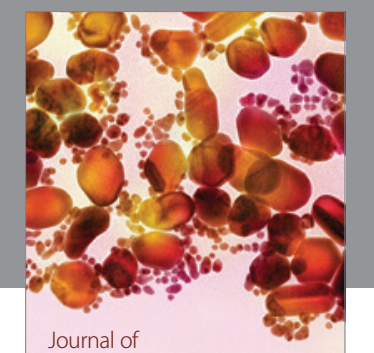

Soft Matter
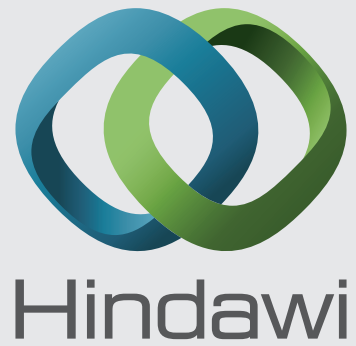

Submit your manuscripts at

http://www.hindawi.com
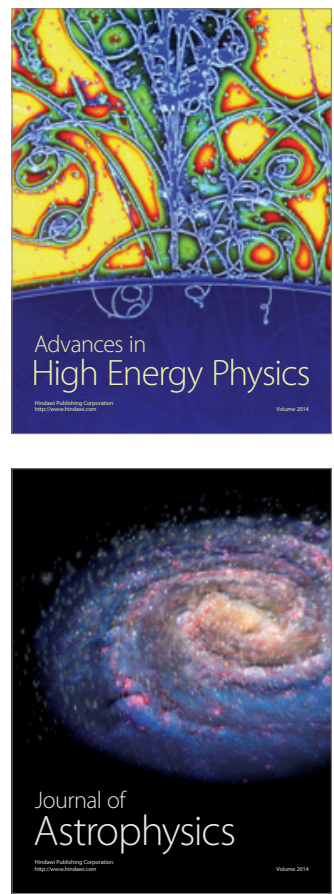
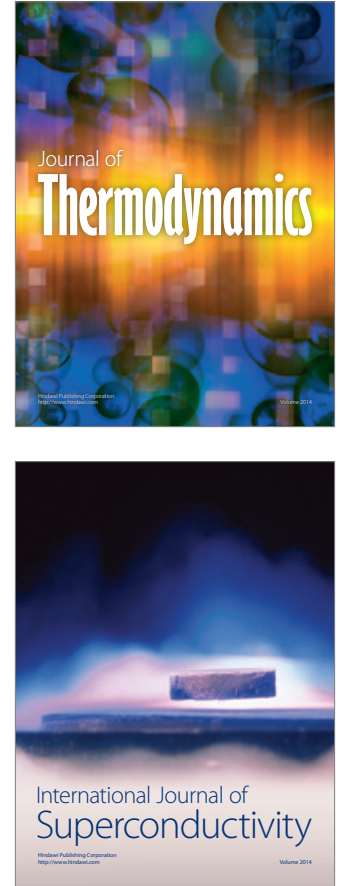
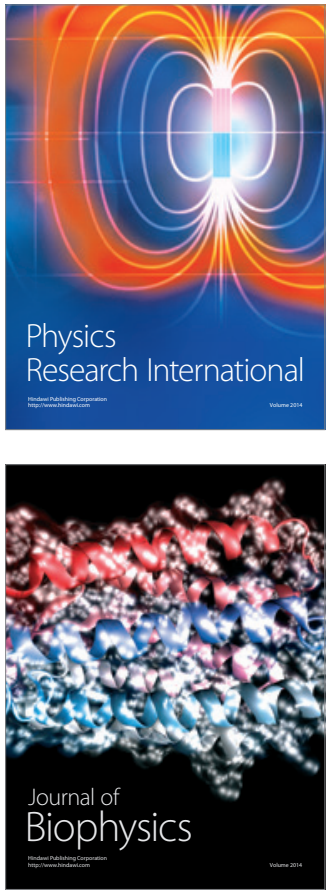
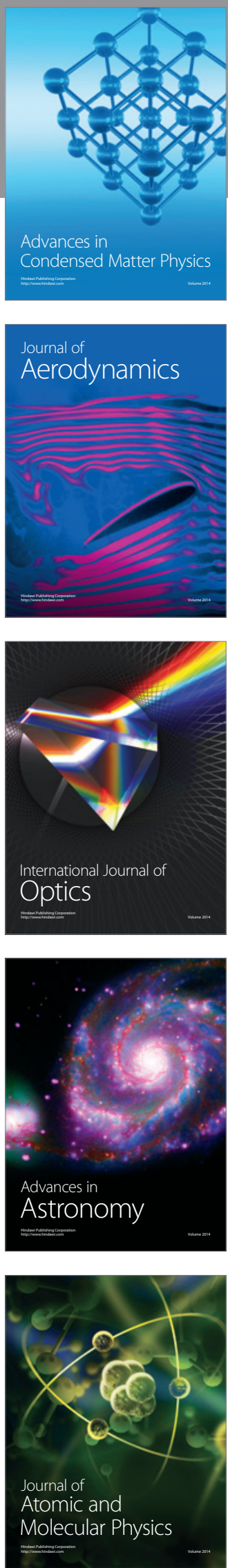